\title{
Structure and Properties of Coordination Polymers Containing Hydrogen-Bonding Capable and Conformationally Flexible Dipyridyl Ligands: An Introductory Undergraduate Research Program at Lyman Briggs College at Michigan State University Robert Laduca, Michigan State University
}

Lyman Briggs College (LBC) is a residential community at Michigan State University for the study of science and its impact on society. The undergraduate-only LaDuca group consisting of LBC students and external REU students has been investigating the synthesis, structure, and properties of coordination polymers containing hydrogen-bonding capable and conformationally flexible dipyridylamide ligands. Hydrothermal reaction of divalent metal nitrates, pyromellitic acid $\left(\mathrm{pymH}_{4}\right)$, and either 1,6hexanediaminebis(nicotinamide) (hbn) or 1,6-hexanediaminebis(isonicotinamide) (hbin) afforded crystalline coordination polymers whose dimensionality depends synergistically on the dipyridylamide nitrogen donor disposition and metal coordination environment. These new phases were structurally characterized via single-crystal X-ray diffraction. $\left[\mathrm{Cd}\left(\mathrm{pymH}_{2}\right)(\mathrm{hbn})\left(\mathrm{H}_{2} \mathrm{O}\right)_{2}\right]_{n}(\mathbf{1})$ and $\left[\mathrm{Co}\left(\mathrm{pymH}_{2}\right)(\mathrm{hbn})\left(\mathrm{H}_{2} \mathrm{O}\right)_{2}\right]_{n}(2)$ display isostructural $(4,4)$ grid topologies with $\left[\mathrm{M}\left(\mathrm{pymH}_{2}\right)\left(\mathrm{H}_{2} \mathrm{O}\right)_{2}\right]_{n}$ chains pillared by hbn ligands. $\left\{\left[\mathrm{Cu}_{2}(\text { pym })(\mathrm{hbn})\left(\mathrm{H}_{2} \mathrm{O}\right)_{2}\right] \cdot 2 \mathrm{H}_{2} \mathrm{O}\right\}_{n}(3)$ manifests a $3 \mathrm{D} 3,4$-connected network with a rare $\left(8^{3}\right)_{2}\left(8^{6}\right)$ topology (pictured) built from the hbn pillaring of $\left[\mathrm{Cu}_{2}(\mathrm{pym})\left(\mathrm{H}_{2} \mathrm{O}\right)\right]_{n}$ layer motifs. $\left\{\left[\mathrm{Zn}_{2}(\text { pym })(\text { hbin })_{2}\left(\mathrm{H}_{2} \mathrm{O}\right)\right] \cdot \mathrm{H}_{2} \mathrm{O}\right\}_{n}(4)$ shows a $1 \mathrm{D}$ ribbon motif based on $\left[\mathrm{Zn}_{2}(\mathrm{OCCCCO})_{2}\right] 14-$ membered circuits. $\left\{\left[\mathrm{Ni}_{2}(\mathrm{pym})(\mathrm{hbin})\left(\mathrm{H}_{2} \mathrm{O}\right)_{4}\right] \cdot 3 \mathrm{H}_{2} \mathrm{O}\right\}_{n}(\mathbf{5})$ exhibits 3,4-connected layer motifs. Luminescent behavior in $\mathbf{1}$ and $\mathbf{3}$ is ascribed to intra-ligand molecular orbital transitions. These phases were also surveyed for the ability to detect nitrobenzene in ethanol suspension.

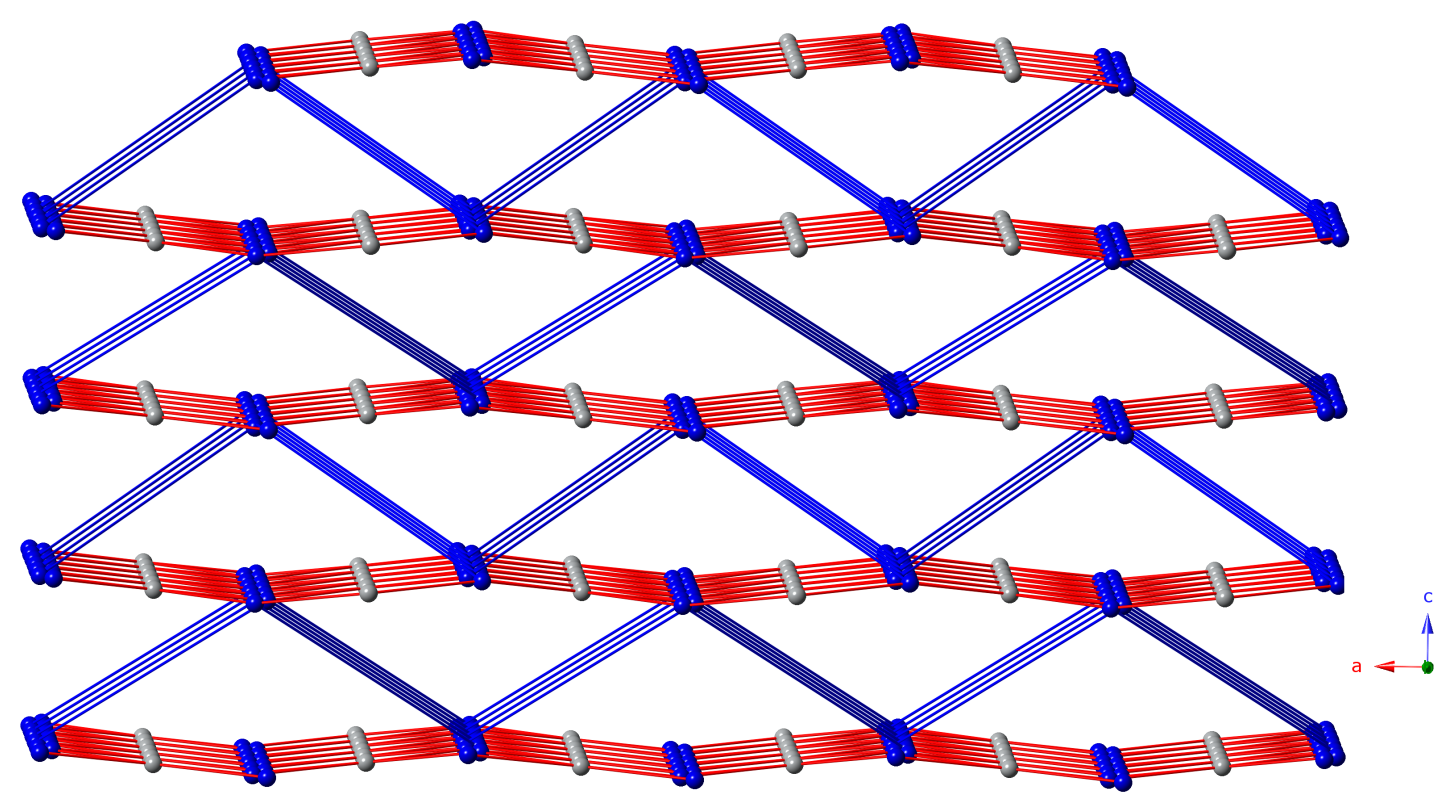

